\title{
Storage Method of Multi-Channel Lidar Data Based on Tree Structure
}

\section{Hao Chen}

Xi'an University of Technology

Fei Gao ( $\nabla$ gaofei@xaut.edu.cn )

Xi'an University of Technology

\section{Qingsong Zhu}

Xi'an University of Technology

\section{Qing Yan}

Xi'an University of Technology

\section{Dengxin Hua}

Xi'an University of Technology

\section{Samo Stanič}

University of Nova Gorica

\section{Research Article}

Keywords: lidar, multi-channel, data storage, tree structure

Posted Date: January 3rd, 2022

DOI: https://doi.org/10.21203/rs.3.rs-1208214/v1

License: (c) (i) This work is licensed under a Creative Commons Attribution 4.0 International License.

Read Full License 


\title{
Storage Method of Multi-channel Lidar Data Based on Tree Structure
}

\author{
Hao Chen ${ }^{1}$, Fei Gao ${ }^{1, *}$, Qingsong Zhu ${ }^{1}$, Qing Yan ${ }^{1}$, Dengxin Hua ${ }^{1}$ and Samo Stanič ${ }^{2}$ \\ 1 Xi'an University of Technology, Xi' an 710048, China; chenhao@xaut.edu.cn (H.C.); 13032920050@163.com (Q.Z.); \\ yanqing@xaut.edu.cn (Q.Y.); xauthdx@163.com (D.H.); \\ 2 University of Nova Gorica, SI-5000 Nova Gorica, Slovenia; samo.stanic@ung.si (S.S.) \\ * Correspondence: gaofei@xaut.edu.cn;
}

\begin{abstract}
The multi-channel lidar has the characteristics of fast acquisition speed, large data volume, high dimension, and strong real-time storage, which makes it difficult to be met using the traditional lidar data storage methods. This paper presents a novel approach to store and convert the multi-channel lidar data by traversal method of the tree structure and binary code. In the proposed approach, a tree structure is constructed based on the multi-dimensional characteristics of multi-channel lidar data and the hierarchical relationship between them. The adjacency table storage structure data in the memory is used to generate the sub-tree of the multi-channel lidar data. The results show that the proposed tree structure approach can save the storage capacity and improve the retrieval speed, which can meet the needs of efficient storage and retrieval of multi-channel lidar data.
\end{abstract}

Keywords: lidar; multi-channel; data storage; tree structure

\section{Introduction}

Lidar, as a new technology of active optical remote sensing detection, has developed rapidly due to its advantages in the profile detection with high temporal and spatial resolutions. It has been used for the remote sensing detection of aerosol particle distribution, atmospheric temperature, humidity, and wind fields, etc. [1].

Lidar makes use of the atmospheric scattering echo signal, which was generated through the interaction of high-power narrow pulse laser with particles and molecules in the atmosphere (such as Mie scattering of aerosols, Rayleigh scattering and Raman scattering of atmospheric molecules, etc.), and was collected by telescope to obtain the height distribution of atmospheric parameters, like atmospheric temperature, humidity, wind velocity, aerosol optical properties based on the inversion method of spectral and energy analyses [2,3]. Elastic scattering lidar, hyperspectral lidar, Raman lidar and differential absorption lidar, as the major detection technologies and methods, play an extremely significant role in the atmospheric remote sensing [4-7]. With the increasing demands of atmospheric remote sensing and environmental monitoring in multi-scale and multi-parameter aspects, lidar tends to develop a comprehensive sensing detection characterized by multiple parameters, long distance, long time, high precision, real time. Therefore, multi-channel lidars that integrate multiple lidar detection technologies is increasingly used for remote sensing detection of atmospheric multi-parameters.

The storage efficiency of detection data is one of the main indicators that affect the performance of the multi-channel lidar system. There are several storage methods of atmospheric lidar data were widely used in recent years, such as character, database, and special format.

The character storage method is the most used storage method. It writes the detection data into the file in text characters and uses separated characters for segmentation. It usually forms a table or sequential structure with the file formats such as CSV [8,9], XLS [10] or TXT $[11,12]$. This method requires high memory capacity and is only suitable for data access operations with a small amount of data. 
The database methods include relational databases (such as Oracle, MySQL, SQL Server, etc.) and time series databases (such as InfluxDB, MongoDB, Cassandra, Couchbase). This method is suitable for large-scale relational data and time series data and is limited in the application of synchronous storage of multi-dimensional data such as spatial-temporal and multi-channel data [13].

The Dedicated Data Storage Method (DDSM) is designed for the lidar data of a specific detection system with the compatibility and scalability limited [14-16].

So, these storage methods are mainly suitable for the lidar data with smaller level, simple data structure and single channel. For the fast storage of multi-channel lidar data, some limitations are presented in these storage methods. In addition, the encoding method of stored files is also an important factor that affects the efficiency of data storage. The above methods mainly use characters or floating-point numbers to store data, which occupy a large amount of memory space, and increases the storage capacity of files and reduces the speed of data retrieval.

To solve the problem of lidar data storage, in the paper, based on the similarity between the multi-channel lidar data structure and tree structure, and their unique characteristics, we investigate a lidar data tree storage structure combining the tree structure data traversal method and the binary encoding of the data, which improves the storage efficiency and reduces the storage space.

\section{Methods}

\subsection{Characteristics of Multi-channel Lidar Data}

At the operation of lidar, a narrow pulse laser beam is emitted from the laser to the atmosphere to interact with the measured parameter target in the atmosphere. Then after the scattered light is received by telescope with splitting and filtering, the laser echo signal is converted into electrical signal for subsequent processing. The lidar equation of single scattering is expressed as follows [17].

$$
P(r)=P_{0} \cdot Y(r) \cdot \frac{c \cdot t_{p}}{2} \cdot \frac{A_{0}}{r^{2}} \cdot \beta(r) \cdot \exp \left[-2 \int_{0}^{r} \alpha\left(r^{\prime}\right) \mathrm{d} r\right],
$$

where $r$ is the detection distance $(\mathrm{m}) ; P(r)$ is the power of echo signal $(\mathrm{W}), P_{0}$ is initial laser power (W); $Y(r)$ is a constant between 0 and 1 , and it is the geometric overlap coefficient of the optical path between the transmitter and the receiver in lidar system $(01) ; c$ is the light speed $\left(3 \cdot 10^{8} \mathrm{~m} / \mathrm{s}\right) ; t_{p}$ is laser pulse width(nm); $A_{0}$ is the aperture area of telescope $\left(\mathrm{cm}^{2}\right) ; \beta(r)$ and $\alpha(r)$ are the atmospheric backscatter coefficient $\left(\mathrm{km}^{-1} \cdot \mathrm{sr}^{-1}\right)$ and extinction coefficient $\left(\mathrm{km}^{-1}\right)$ respectively, which are related with atmospheric conditions.

The intensity of lidar data represents the state information of atmospheric parameters at different detection distances along the lidar direction, which referring to the data structure of atmospheric parameter profiles corresponding to the distance point $r_{i}(i=1,2, \ldots, n, n$ is the total point number along the detection direction) and the intensity value of laser echo signal $p_{i}$. Then the data value of atmospheric parameter at $t_{j}$ can be expressed as

$$
v_{j}=\left\{\left(r_{1}, p_{1}\right)_{j},\left(r_{2}, p_{2}\right)_{j}, \ldots,\left(r_{n}, p_{n}\right)_{j}\right\},
$$

where $j=1,2, \ldots, m, j$ is the index of lidar data and $m$ is the maximum index number. $v_{j}$ is called a lidar data unit (LDU), and each LDU is a group of lidar profile data.

The multi-channel lidar system uses the hyperspectral discriminator to achieve the separation and extraction of specific spectral signals $[18,19]$. The spectral data of laser echo signal is synchronously recorded in each channel, and the multi-channel lidar data includes the data information such as echo signal intensity, detection range, recording time, and channel number, etc. 
So, at $t_{j}(j=1,2, \ldots, m)$ within the $k^{\text {th }}$ channel $(k=1,2, \ldots, q, q$ is the maximum number of data acquisition channels in the multi-channel lidar system), the laser echo signal data at the distance point $r_{i}$ can be expressed as

$$
v_{i, j}^{k}=\left(r_{i}, p_{i}\right)_{j}^{k},
$$

the multi-channel lidar data $V$ can be presented as follows

$$
V=\left(\begin{array}{llll}
v^{1} & v^{2} & \cdots & v^{q}
\end{array}\right)=\left(\begin{array}{cccc}
v_{1}^{1} & v_{1}^{2} & \cdots & v_{1}^{q} \\
v_{2}^{1} & v_{2}^{2} & \cdots & v_{2}^{q} \\
\vdots & \vdots & & \vdots \\
v_{m}^{1} & v_{m}^{2} & \cdots & v_{m}^{q}
\end{array}\right),
$$

then

$$
v_{j}^{k}=\left\{\left(r_{1}, p_{1}\right)_{j}^{k},\left(r_{1}, p_{1}\right)_{j}^{k}, \cdots\left(r_{n}, p_{n}\right)_{j}^{k}\right\},
$$

Each column in data $V$ corresponds to the channel unit of the multi-channel lidar data. The four-dimensional structure of the multi-channel lidar data is shown in Figure 1 .

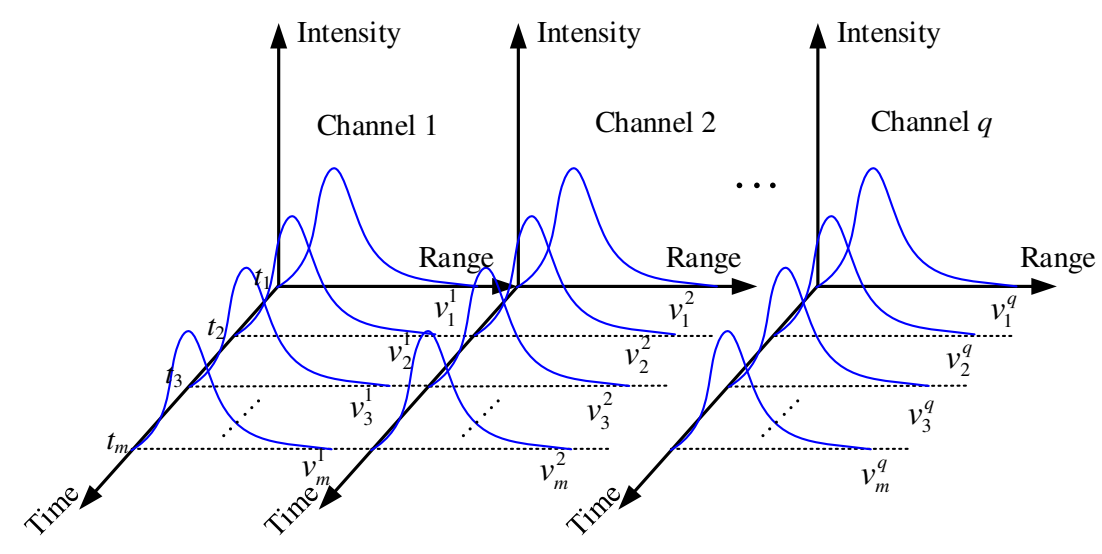

Figure 1. Four-dimensional structure of the multi-channel lidar data

From the above analyses, we know that the multi-channel lidar data is consist of several single channel lidar data. Compared with the single-channel lidar data, the channel dimension information is added to the multi-channel lidar data. It can get an LDU in each channel of the multi-channel lidar data, and each LDU has a channel-time relationship. Therefore, the multi-channel lidar data have a large amount of data and a complex structure, and the storage methods of single-channel lidar systems are not applicable to the multi-channel lidar data.

\subsection{Tree Structure of Lidar Data Storage}

\subsubsection{Tree Structure}

Tree structure is a typical nonlinear data structure with multi-level nested relationship, which is often used to represent the data set with the characteristics of "one to many" relationship [20].

As shown in Figure 2, tree is a limited data set composed of $h(h>0)$ nodes. The first node of the tree is called the root, and nodes without children are called leaf. The intermediate node between the root and the leaf nodes is called an internal node. When $h>1$, the remaining nodes of the tree can be regarded as multiple disjoint finite sets, and each set can be regarded as a subtree of the root. The tree structure can classify and sort data effectively, and each node 
has a unique address. The subtrees are independent of each other, and the operations of the subtree do not affect each other.

The tree structure can clearly express the relationship between data with multi-level and multi-category attributes, and can organize data with the complex relationship efficiently.

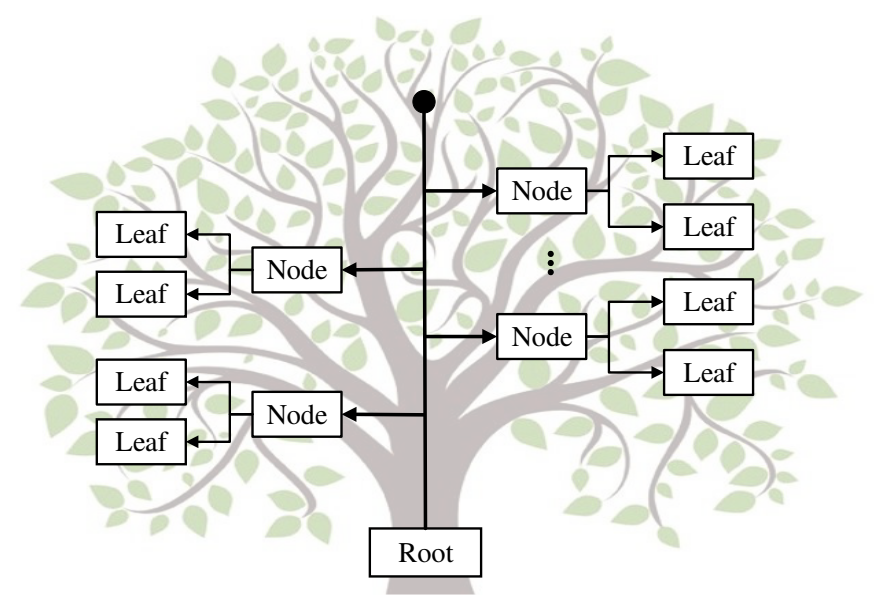

Figure 2. Tree structure

\subsubsection{Tree structure of multi-channel lidar data (TSMLD)}

There is a four-dimensional relationship (such as channel, time, range, intensity) between multi-channel lidar data, and its arbitrary data $v_{i, j}^{k}$ shows different hierarchical distribution in different dimensions.

In general, the multi-channel lidar collects data synchronously from all channels in time. However, the spectral information of laser echo signals and the properties of data in different channels are different.

As shown in Figure 1 , at $t_{1}, t_{2}, \ldots, t_{m}, q$ channels obtain $q$ sets of data synchronously, and each set of data can draw a profile with laser echo intensities. The four-dimensional structure of lidar is similar to the tree structure with the origin of the coordinate system as the root node shown Figure 2, also including the root node Root, branch nodes Node and leaf nodes Leaf.

To show the hierarchical relationship of multi-channel lidar data more clearly, virtual node sets such as root node, detection time node set, and channel node set are introduced, as shown in Figure 3. The nodes from the first to the third level in Figure 3 are virtual nodes (the gray node). The nodes from the fourth to the last level are the multi-channel lidar data set, which represents the detection data from $r_{1}$ to $r_{n}$ (the white node). Given the first-level node of the tree structure of multi-channel lidar data be the root node of the tree, denoted as $D$, corresponding to the Root node, and the second-level nodes are the detection time node sets, denoted as $T$, the third-level nodes are the detection channel node sets, denoted as $C$, and the other level nodes are the laser echo data node sets, denoted as $v$. The second-level and the third-level are branch nodes of tree, corresponding to the Node nodes. The detection data nodes from $r_{1}$ to $r_{n}$ together form the leaf nodes of the tree, corresponding to the Leaf nodes. 


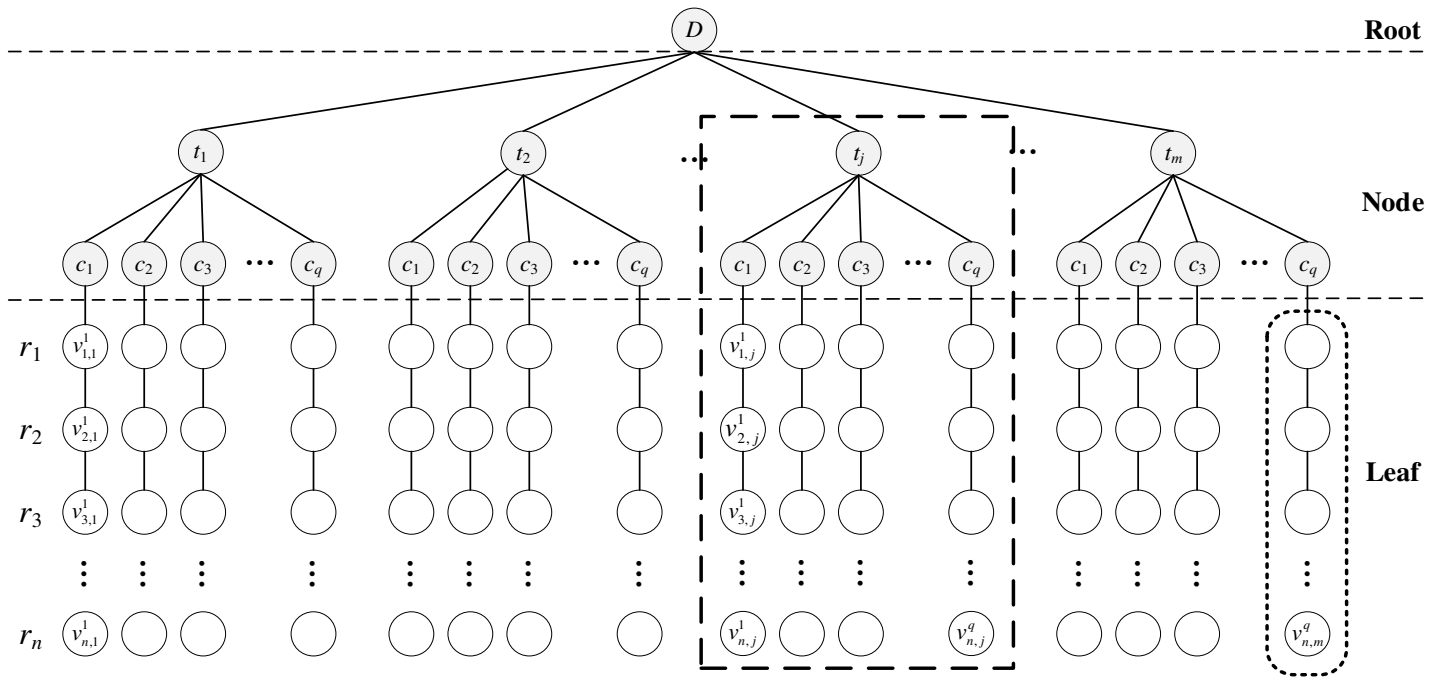

Figure 3. Tree structure of multi-channel lidar data

The TSMLD shown in Figure 3 is a connected acyclic undirected graph, denoted as $G$, $G=\left\{G_{1}, G_{2}, G_{3}, \ldots, G_{q}\right\}, G_{j} \in G, 1 \leq j \leq m, G_{j}$ is the subtree of detection time in $G$, representing the data of all channels at the $j^{\text {th }}$ detection time. The subtree $G_{j}$ is shown in the dotted box in Figure 3.

\subsubsection{The adjacency list of TSMLD}

The adjacency list is the common storage method of graph. Based on the hierarchical relationship of the tree structure $G$ of the multi-channel lidar data and the structure of the linked table and the array, the paper uses adjacency list structure to represent the storage structure of the multi-channel lidar data. The array based on continuous memory space is used to store a small number of nodes, such as root node, detection time node and channel node. The linked list based on distributed storage space is used to store a large number of detection data nodes. The node of tree structure can be retrieved quickly by using the subscript of the array and the address of the linked list [21,22]. Therefore, the adjacency list structure of the subtree $G_{j}$ is constructed as shown in Figure 4.

The root node of the adjacency list of $G_{j}$ is expressed as the detection time node $t_{j}$, and the sub-node of node $t_{j}$ is the detection channel node set. The sub-node of the detection channel node $c_{k}$ is the multi-channel lidar echo data set, which represents the intensity value of detection data. For the adjacency list of tree structure $G$ of multi-channel lidar data, the root node $D$ is created and the subtrees $G_{1}, G_{2}, G_{3}, \ldots, G_{m}$ is added to the sub-nodes of node $D$. The continuous spatial storage is used to deal with the nodes $t_{1}, t_{2}, t_{3}, \ldots, t_{m}$ of $G_{1}, G_{2}, G_{3}, \ldots, G_{m}$.

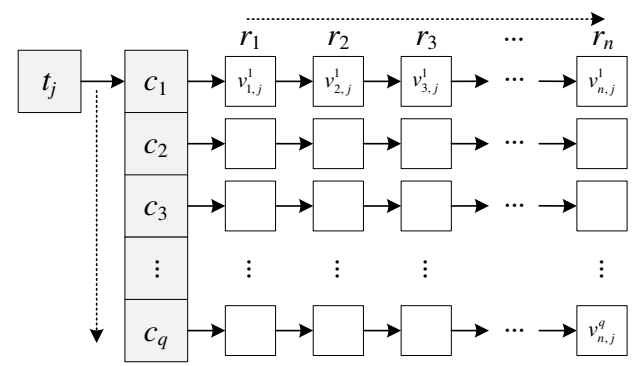

Figure 4. The adjacency list structure of the subtree $G_{j}$

Therefore, the generation procedure for the adjacency list of the tree structure $G$ of the multi-channel lidar data is described as follows. To save memory space, we represent the detection data by binary code.

(1) Declare an array of type Time ajtime $[m]$, let ajtime $[m]=\left\{t_{1}, t_{2}, t_{3}, \ldots, t_{m}\right\}$;

(2) Declare an array of type Channel ajchannel[ $q]$, let ajchannel $[q]=\left\{c_{1}, c_{2}, \ldots c_{q}\right\}$. 
(3) Connect the addresses of the array according to the structure of the subtree $G_{j}$.

The algorithm is shown in Table 1.

Table 1 The generation algorithm for the adjacency list of $G$

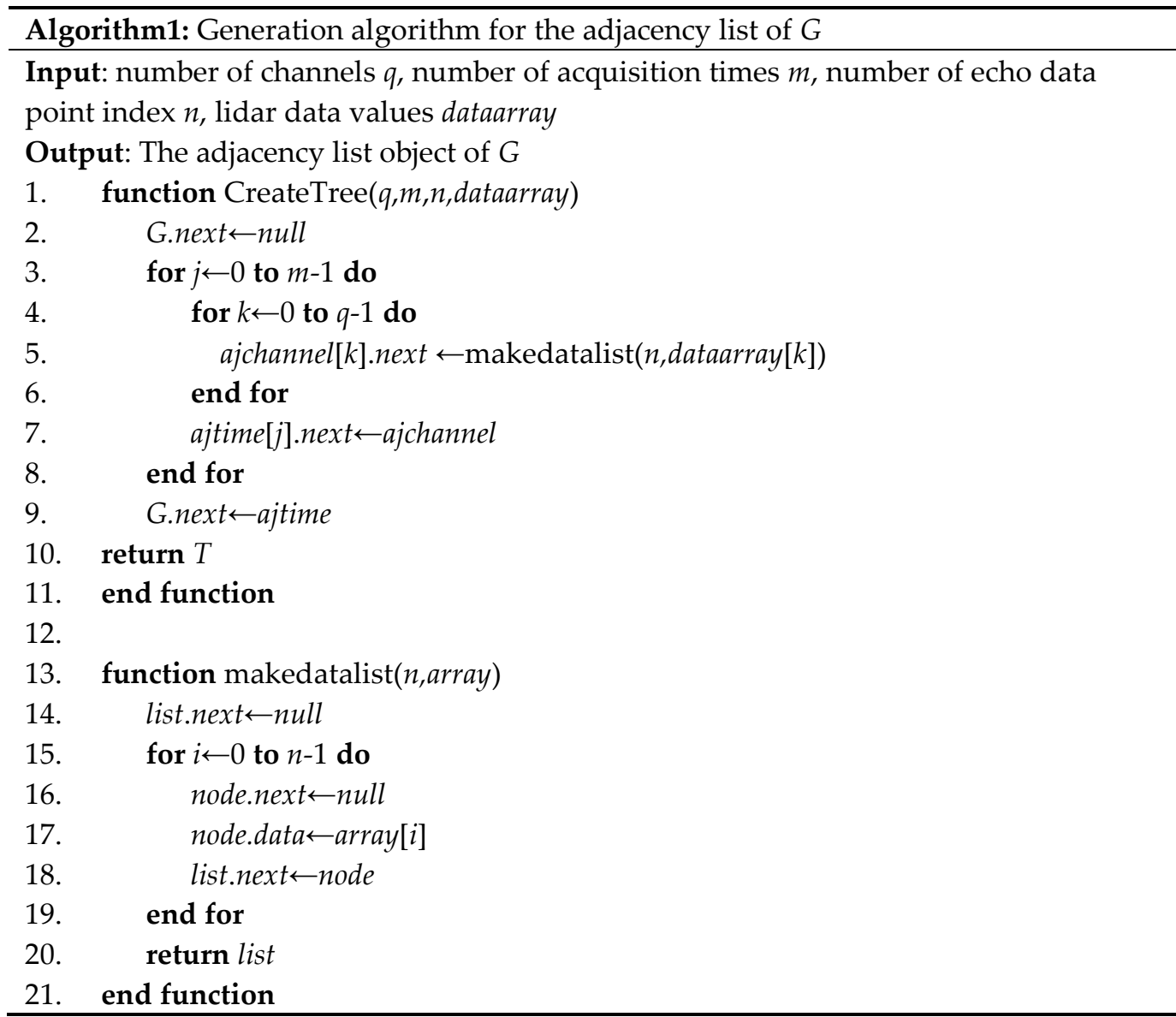

The total number of nodes for each multi-channel lidar data is len $=q^{*} m^{*} n$. The time complexity of the generation algorithm for the adjacency list of TSMLD is O(len), that is, each data node needs to be accessed once.

Then, the tree structure of the multi-channel lidar data stored by binary code in the adjacency list is converted into a data file, which is coded by binary using the traversal method of tree structure.

\subsubsection{Binary format of TSMLD storage files}

Binary formats offer advantages in terms of speed of access. While the basic unit of information is very straightforward in a data file stored in characters (one byte equals one character), finding the actual data values is often much harder. This means that it is usually necessary to read the entire file to find any value [23-25].

For binary files, some sort of format description, or map, is required to be able to find the location of any value in the file. However, the advantage of having such a map is that any value within the file can be found without having to read the entire file.

In addition, in terms of memory, binary files stored data using numeric formats rather than as characters, tends to use less memory.

To save the storage space and improve the retrieval efficiency of TSMLD, we present a binary coding file structure of TSMLD. The binary coding file can store many tree structure $G$, so, its structure includes some header information, both for the overall file, and for subsections within the file. This header information contains information such as follows:

(1) The number of the tree structure $G$. 
(2) The beginning tag for $G$.

(3) The file size.

(4) The number of bytes used for each data value (the data value size).

(5) The byte location within the file where a set of TSMLD values begins (a pointer).

After the header information, some TSMLDs are stored in tree segment. Each tree segment is a tree structure $G$, and have a header which includes some information such as the number of detection time nodes, the data size (the length of data segment) and the tree segment number. The back part of the tree segment is data segment, which contains the data node set of the tree structure $G$.

Each data segment is a subtree $G_{j}$, and have a header includes the number of channel nodes $q$, the data size (the length of sub segment) and the data segment number. Behind of the header of data segment is some sub segments.

There are $n$ Leaf nodes in each sub segment, and each sub segment is consisting of $n$ echo data values and sub segment header. Similarly, the sub segment header information contains the length of data value and the record number.

The binary file structure of TSMLD is shown as Figure 5 .

File Structure
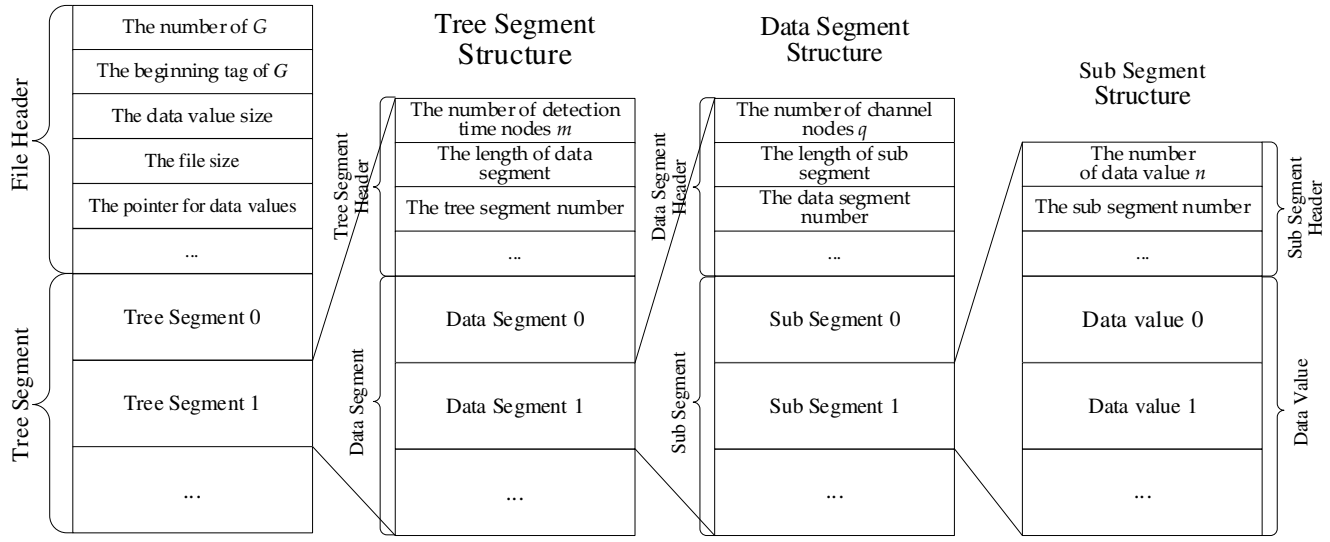

Figure 5. The binary file structure of TSMLD.

\subsubsection{Storage of TSMLD}

In each detection experiment, the data collected from all channels in the multi-channel lidar system constitute a tree structure object of detection time sequence. A tree data storage method (TDSM) of TSMLD is given as follows.

To achieve TSMLD, the tree structure $G$ must be traversed firstly. According to the structure characteristics of the tree structure $G$, the traversal methods can be divided into two ways: time-first storage (TFS) and channel-first storage (CFS) [26-28]. The TFS method preferentially stores the data collected by each channel at the same detection time, and the CFS method preferentially stores the data collected by each detection time at the same detection channel. The data sequence of the TFS method and the CFS method is shown in Figure 6.

\begin{tabular}{|l|l|l|l|l|l|l|l|l|l|l|l|l|l|l|}
\hline$v_{1}^{1}$ & $v_{1}^{2}$ & $v_{1}^{3}$ & $\ldots$ & $v_{1}^{q}$ & $v_{2}^{1}$ & $v_{2}^{2}$ & $v_{2}^{3}$ & $\ldots$ & $v_{2}^{q}$ & $v_{3}^{1}$ & $v_{3}^{2}$ & $v_{3}^{3}$ & $\ldots$ & $v_{3}^{q}$ \\
\hline
\end{tabular}

(a)

\begin{tabular}{|l|l|l|l|l|l|l|l|l|l|l|l|l|l|l|}
\hline$v_{1}^{1}$ & $v_{2}^{1}$ & $v_{3}^{1}$ & $\ldots$ & $v_{m}^{1}$ & $v_{1}^{2}$ & $v_{2}^{2}$ & $v_{3}^{2}$ & $\ldots$ & $v_{m}^{2}$ & $v_{1}^{3}$ & $v_{2}^{3}$ & $v_{3}^{3}$ & $\ldots$ & $v_{m}^{3}$ \\
$\ldots$
\end{tabular}


Figure 6. The data sequence of traversal methods. (a) the data sequence of TFS; (b) the data sequence of CFS.

The detection time information of multi-channel lidar data is given priority in the TFS method, therefore, the multi-channel lidar data is stored in the order of detection time. The data acquired at a certain detection time is appended to the data acquired at the previous detection time, and the final data storage sequence is $\left\{v_{1}^{1}, v_{1}^{2}, \ldots, v_{1}^{q}, v_{2}^{1}, v_{2}^{2}, \ldots, v_{2}^{q}, v_{3}^{1}, v_{3}^{2}, \ldots, v_{3}^{q}, \ldots\right\}$. The detection channel information of multi-channel lidar data is given priority in the CFS method, and the multi-channel lidar data is stored in the order of detection channel. Therefore, the data acquired in each detection channel is appended to the data acquired in the previous detection channel, and the final data storage sequence is $\left\{v_{1}^{1}, v_{2}^{1}, \ldots, v_{m}^{1}, v_{1}^{2}, v_{2}^{2}, \ldots, v_{m}^{2}, v_{1}^{q}, v_{2}^{q}, \ldots v_{m}^{q}\right.$ ...\}.

The set of multi-channel lidar data $\left\{v_{j}^{1}, v_{j}^{2}, \ldots, v_{j}^{q}\right\}(j=1,2, \ldots, m)$ on detection time $t_{j}$ is consistent with the minimum data storage unit obtained by a single-time multi-channel lidar. In the CFS method, both channel and node tags need to be added to the stored data for data splitting, while only node tags need to be added in the TFS method. So, in the process of data storage and reading, both above methods require additional operation tags to address or split the data, which leads to many redundant operations and reduces storage efficiency. In this paper, the cache storage mechanism is introduced to combine the TFS with the CFS methods. By establishing the cache space, the TSMLD is converted and stored to a data file and the data coded by binary. The process of conversion and storage is shown in Figure 7.

The main steps of conversion and storage method are described as follows:

(1) Read the binary-coded data $\left\{v_{j}^{1}, v_{j}^{2}, \ldots, v_{j}^{q}\right\}$ at time $t_{j}$ by time sequence, and write to the cache container buffer[q].

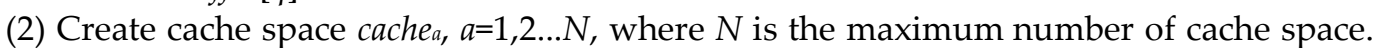
Write buffer $[q]$ to cachea in units of detection time by TFS. Let $l$ be the maximum length of cache $a$, then, cache $e=\mathrm{U}_{j=0}^{j=l}\left\{v_{j}^{1}, v_{j}^{2}, \ldots, v_{j}^{q}\right\}$.

(3) If the cachea is full, create cache space cachea+1, and repeat steps (1) and (2) until data collection completed.

(4) Create an array ldsArray $[q][l]$, read cachea row by row, and store the row data into ldsArray $[q][l]$ by CFS.

(5) Write $l d s$ Array $[q][l]$ to the data file File coded by binary, and add some header information to the data file, then let $a=a+1$, and back to step (4).

(6) When a $>N$, the TFS and CFS are integrated to store the data cache space.

(7) Close the File and clear all cache space.

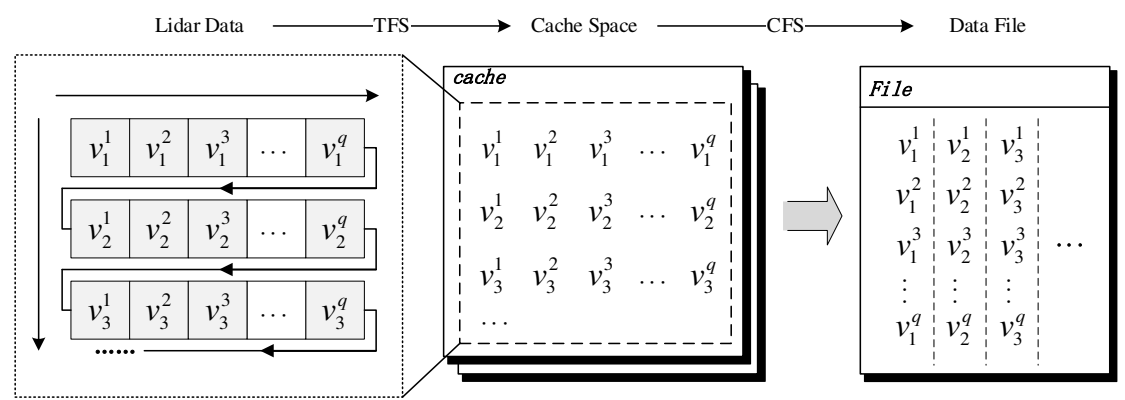

Figure 7. Process of conversion and storage of TSMLD

The main process of conversion and storage method is shown in Figure 8. 


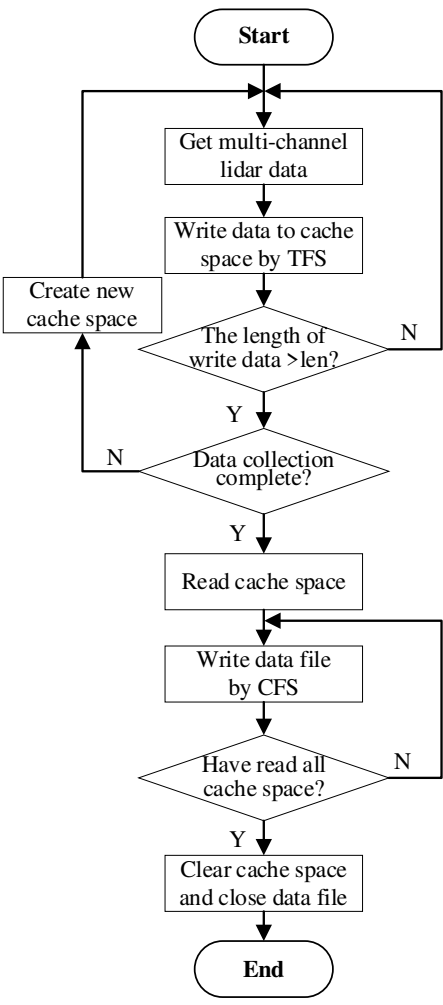

Figure 8. The process of conversion and storage method.

By reading the multi-channel lidar data storage files, arbitrary detection data can be retrieved according to the number of channels and time sequence.

Due to the multi-channel lidar data storage files are encoded in binary, so we can get the detail information by the fix-length byte and the structure of the data file shown in Figure 6 . Then, any data value can be read by the definition of header information for the data segments, and the data file can be read quickly by row or column of data file. The data retrieval process is shown in Figure 9.

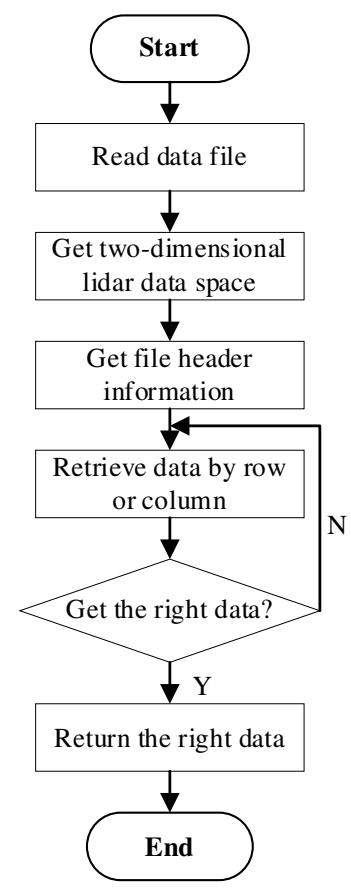

Figure 9. The process of data retrieval 


\section{Experimental}

The experimental data comes from the ultraviolet Raman lidar system in the Center for Lidar Remote Sensing Research of Xi'an University of Technology [29]. In the experiment, an industrial control cabinet Pxie-1071 and a data acquisition card Pxi-5105 shown in Figure 10 (a) and (b), developed by NI company, are used as data acquisition equipment, with the main parameters shown in Table 2, which are suitable for the data acquisition in the multi-parameter integrated detection lidar system. The storage and retrieval experiment for lidar echo data is performed under the multi-channel mode, and the hardware system and the user interface of the software system for data acquisition is shown in Figure $\mathbf{1 1 .}$

Table 2. Main parameters in the experimental platform

\begin{tabular}{cc}
\hline Parameters of data acquisition card & Value \\
\hline Channel number of single acquisition card & 8 \\
Maximum real-time sampling rate & $60 \mathrm{MS} / \mathrm{s}$ \\
Signal input range & $0-30 \mathrm{~V}$ \\
Built-in memory of board card & $512 \mathrm{MB}$ \\
Maximum single acquisition length (8-channel mode) & 16384 \\
CPU model & $\mathrm{i} 5-4200$ \\
Main frequency of CPU & $2.3 \mathrm{GHZ}$ \\
Disk space & $48 \mathrm{G} \mathrm{SSD}$ \\
Built-in memory & $8 \mathrm{~GB}$ \\
Maximum system bandwidth & $3 \mathrm{~GB} / \mathrm{s}$ \\
\hline
\end{tabular}

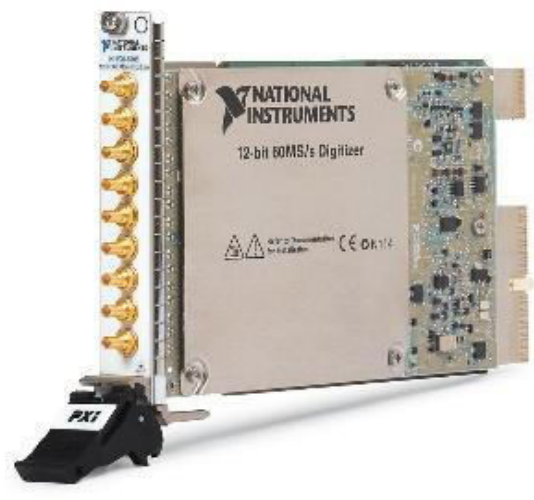

(a)

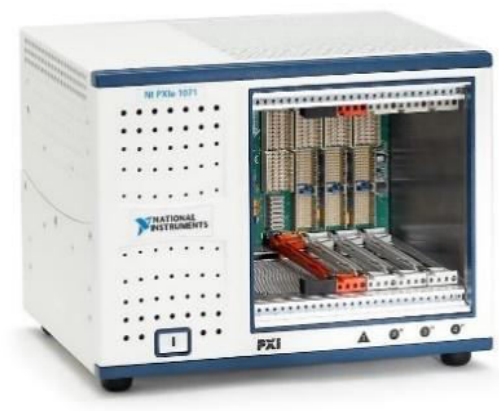

(b)

Figure 10. Data acquisition card and industrial control cabinet. (a) Data acquisition card Pxi-5105 ; (b) Industrial control cabinet Pxie-1071 


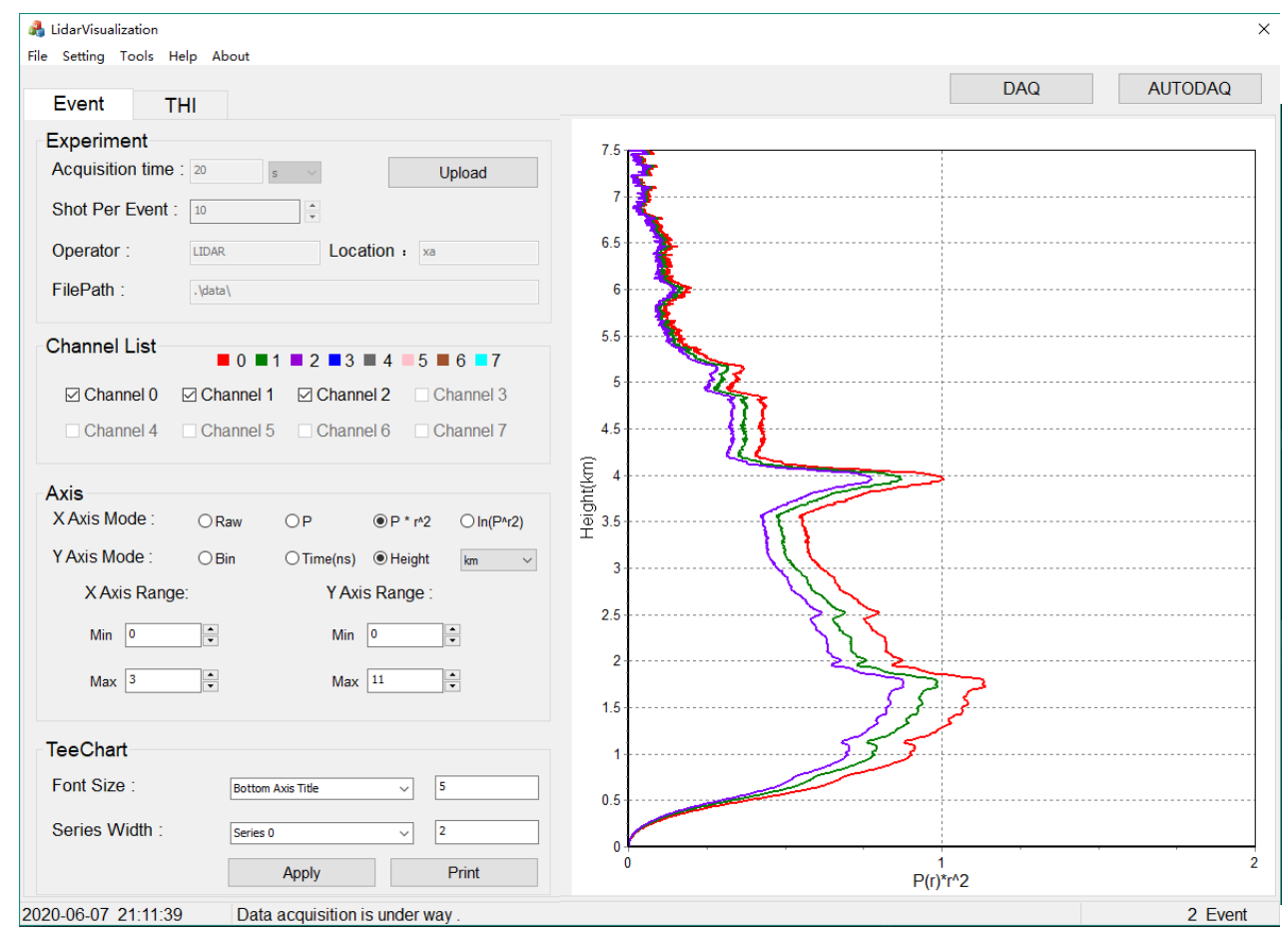

Figure 11. Interface of software system of data acquisition

\section{Results}

In the storage capacity test, we consider four storage methods, such as two structure of character storage method, the table structure storage method (TSSM) and text sequence storage method (TSM), the database storage method (DSM), the tree data storage method (TDSM) given in this paper. An elaborate comparison of these four storage methods is conducted in terms of the storage capacity and retrieval speed of the multi-channel lidar data. The TSSM, TSM, DSM and TDSM are stored in CSV file, text file(TEXT), MySQL database and binary file, respectively.

Figure 12 presents the variation of the file storage capacity of the multi-channel lidar echo data with the four storage methods.

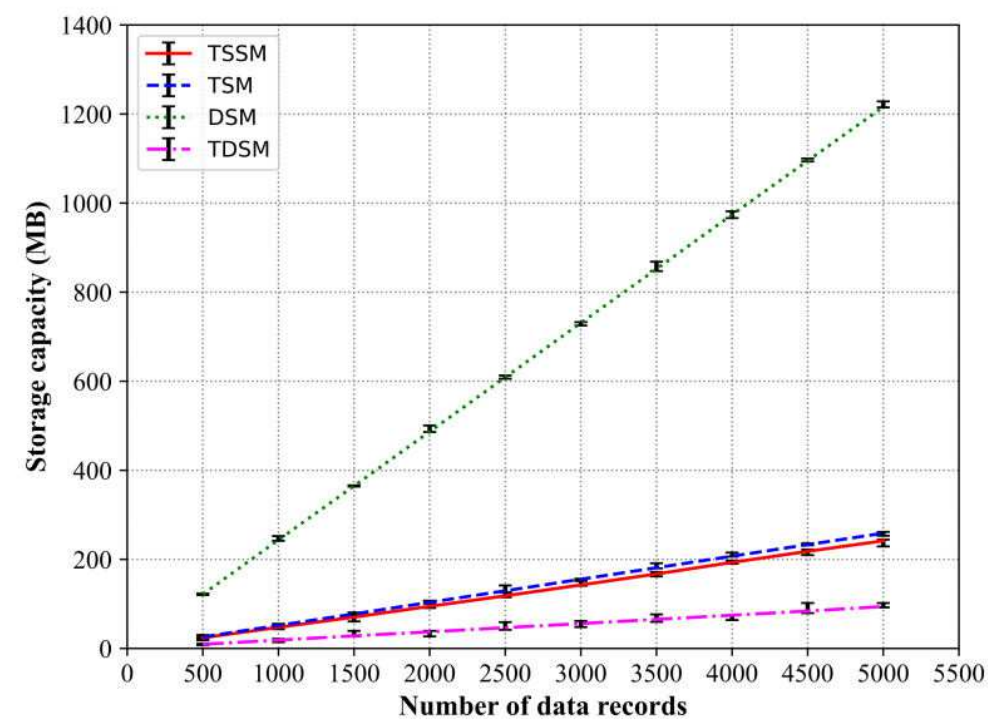

Figure 12. Comparison of storage capacities between TSSM, TSSM, DSM and TDSM 
As Figure 12 shows, with the increase of multi-channel lidar data, the storage capacities of TSM and TSSM are almost the same and increase linearly, because both are character-based storage methods which have the fixed storage capacity in every character. The DSM has the largest storage capacity because the structured approach is utilized to improve the retrieval speed in MySQL database system, but the building of data indexes in the relational model results in the multiplied increase of storage space. With the same data volume, the TDSM has the minimum storage capacity owing to the compressibility of binary storage method in comparison to the text character method. The text character method focuses on the distribution of storage space for each character, while the binary data aims to compress and store all the data into a more compact file with more space saved in the meanwhile.

Figure 13 shows the storage capacity reduction rate of the TDSM compared with the TSSM, TSM and DSM. The TSSM and TSM have similar trends in the reduction rate of storage capacity, ranging from $60 \%$ to $65 \%$. However, the DSM with the maximum storage capacity has a significant reduction rate of about $92 \%$.

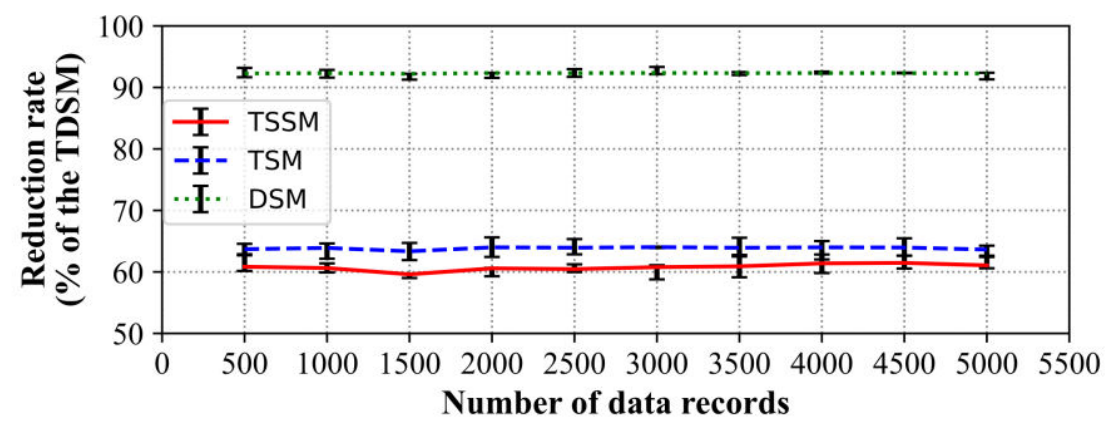

Figure 13. The reduction rate of storage capacity of TSSM, TSSM,DSM and TDSM

In the retrieval speed test, we mainly test the multi-channel lidar data retrieval speed of four methods for 1000 random retrieval visits under different data volumes, and the test result is shown in Figure 14.

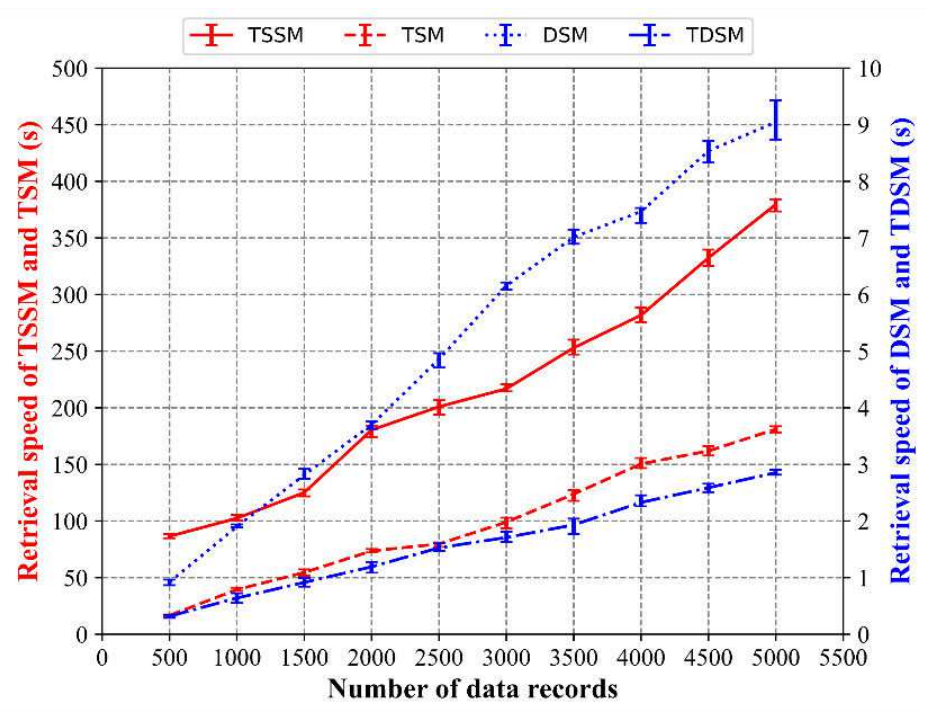

Figure 14. Comparison of data retrieval speed between TSSM, TSSM, DSM and TDSM

From Figure 14, we can find that the TSSM is the most time-consuming method, followed by the TSM, and the time consumption of DSM and TSDM methods is kept at a low level with the least time of less than 10 seconds. With the increase of multi-channel lidar data, there is a linear increase in the data retrieval time of TSSM and TSM methods since a linear increase is 
also shown in the data storage capacity and the data retrieval is linearly correlated with the data volume. Similarly, the TSSM, with the increase of multi-channel lidar data, is affected by the reading and writing speed of $\mathrm{I} / \mathrm{O}$ and the retrieval speed of characters. It leads to an obvious reduction of retrieval speed and the increase of time consumption. Based on the professional database management system, the DSM uses the structured approach to deal with field data and create indexes for field data. Despite the increase of data storage space, an obvious optimization effect is shown in the improvement of data retrieval efficiency. The time of data retrieval of the DSM and the TDSM is less than 10 seconds, and the TDSM takes less than 5 seconds in the experimental test. Due to a combination of the tree structure traversal method and binary coding, the data at any position in the data file can be quickly read based on the detection time and the channels. The method is less affected by the amount of data, and it saved the time of data retrieval. In other words, this method reduces the time consumption of multi-channel lidar data storage. In addition, the large amount of multi-channel lidar data need less memory to be the buffer during storage.

By Comparing with the TSSM, TSM and DSM, Figure 15 shows the reduction rate of data retrieval time based on TDSM. It turns out that the reduction rate of TDSM reaches $98 \%$ because of the obvious improvement of retrieval efficiency compared to the TSSM and TSM.

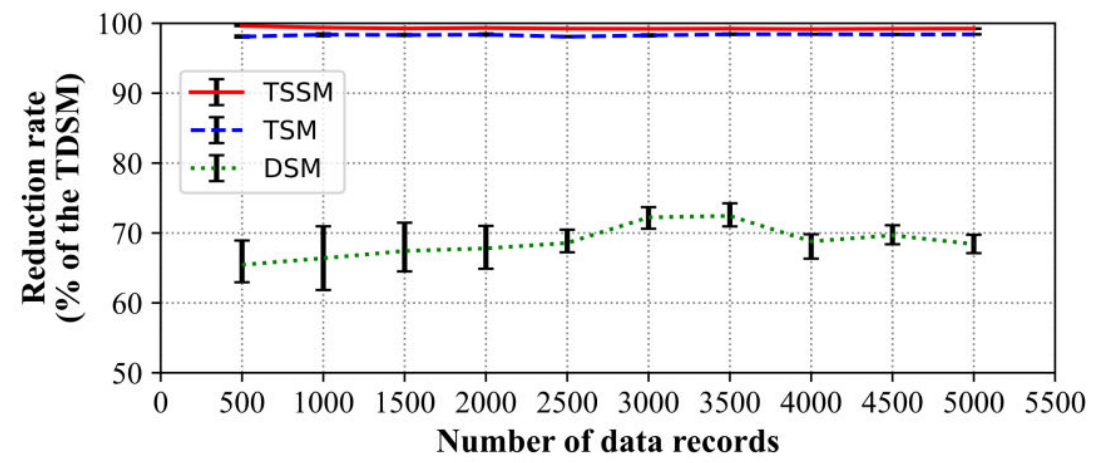

Figure 15. The reduction rate of data retrieval time of TSSM, TSSM, DSM and TDSM

In addition, the data retrieval time of the TDSM and DSM is kept at a relatively low level, and the retrieval time is reduced by about $70 \%$, fluctuating between $65 \%$ and $72 \%$ compared with the DSM.

\section{Discussion}

The software system for data acquisition of multi-channel lidar system integrates the method proposed in this paper, and the programming language is Visual Studio C++ 2010. Due to technical limitations, it can only run-on windows series operating system. The operating system used in the experiment is Windows 10. However, a full multi-channel lidar system contains multiple functional subsystems. The control subsystem is used to control the hardware devices in the multi-channel lidar system, and it usually runs on Linux systems such as Ubuntu and Debian. If the data acquisition system and the control software system can be integrated and run across platforms, the work efficiency of the multi-channel lidar system can be further improved. The cross-platform operation of the data acquisition system requires drivers for different operating systems to connect to the data acquisition card and the different programming language or software framework is used to program the data acquisition system. But the replacement of operating systems and programming languages will inevitably affect the performance of the data acquisition system. How to be affected or to be affected by what factors, that will be studied in the next research work.

\section{Conclusion}

Through the analysis of relational characteristics and storage requirements for lidar data, the present paper develops a storage method of the multi-channel lidar data based on the tree 
structure for the multi-channel lidar system. Drawing on the hierarchical relationship structure of channel, time, and range of multi-channel lidar data, this method combined the linked list and the adjacency list with an array structure to construct the storage method, and the multi-channel lidar data is encoding by binary code in the adjacency list. Finally, the multi-channel lidar data is stored in the binary format files. This study can be used to build data processing and storage systems for the multi-channel lidar system or similar system. In addition, it can be an example of a solution to similar lidar system when a selection from a list of alternatives is required. The experimental results show that this method, compared with the traditional list structure and the text character storage method, can save at least $60 \%$ of the storage capacity and increase the retrieval speed by about $98 \%$. The superior advantages of the method lay a solid foundation for the effective use of multi-channel lidar data.

\section{Author information}

\section{Affiliations}

Xi'an University of Technology, China

Hao chen,Fei Gao, Qingsong Zhu, Dengxin Hua

University of Nova Gorica, Slovenia

Samo Stanič

\section{Corresponding author}

Correspondence to Fei Gao.

\section{Authors' Contributions}

Conceptualization, H.C.; methodology, H.C.; formal analysis, F.G.; writing-original draft preparation, Q.Z.; data curation, Q.Y.; supervision, D.H and S.S. All authors have read and agreed to the published version of the manuscript.

\section{Funding}

This research was funded by NSFC (No. 61805194, No.41775035), Natural Science Basic Research Plan in Shaanxi Province of China (No.2019JQ-019) and China-CEEC Higher Education Institutions Consortium (No. 202017), Innovating Capability Support Program of Shaanxi (No. 2019 GHJD-09).

\section{Availability of data and materials}

All materials and data used should be available at Xi'an University of Technology/China.

The data used to support the findings of this study are available from the corresponding author upon request.

\section{Competing interests}

The authors have declared no conflict of interests for this research.

\section{Acknowledgments}

We thank the financial supports of the Intergovernmental Scientific and Technological Cooperation Project between China and Slovenia (2021-1-16) supported the academic exchange between the University of Nova Gorica and Xi'an University of Technology.

\section{References}

1. Mole M, Wang L, Stanič S, Bergant K, Eichinger WE, Ocaña F, Strajnar B, Škraba P, Vučković M, Willis WB (2017) Lidar measurements of Bora wind effects on aerosol loading. Journal of Quantitative Spectroscopy and Radiative Transfer 188:39-45

2. Yabuki M, Matsuda M, Nakamura T, Hayashi T, Tsuda T (2016) A scanning Raman lidar for observing the spatio-temporal distribution of water vapor. Journal of Atmospheric and Solar-Terrestrial Physics 150:21-30 
3. Shen F, Cha H, Sun D, Kim D, Kwon SO (2008) Low tropospheric wind measurement with Mie Doppler lidar. Optical review 15(4):204-209+

4. Kotsakis A, Robinson J, Sullivan J, Berkoff T, Gronoff G, Knepp T, Cede A, Swap R (2019) Comparison and spatiotemporal analysis of ozone from Pandora, ozonesonde, and ozone lidar measurements during OWLETS. In: Geophysical Research Abstracts

5. Yan Q, Wang Y, Gao T, Gao F, Di H, Song Y, Hua D (2019) Optimized retrieval method for atmospheric temperature profiling based on rotational Raman lidar. Applied optics 58(19):5170-5178

6. Baars H, Seifert P, Engelmann R, Wandinger U (2017) Target categorization of aerosol and clouds by continuous multiwavelength-polarization lidar measurements. Atmospheric Measurement Techniques 10(9):3175-3201

7. Zheng J, Sun D, Chen T, Dou X, Zhao R, Li Z, Zhou A, Zhang N, Gao J, Wang G (2018) Wind profiling from high troposphere to low stratosphere using a scanning Rayleigh Doppler lidar. Optical Review 25(6):720-728

8. Bo S, Sha-lei S (2012) Implementation of Data Acquisition and Processing System in Multi-Spectral Lidar Based on LabVIEW. Optics \& Optoelectronic Technology 6

9. Wan Y, Yao J, Li W, Li L (2012) Research on data acquisition and processing of laser radar signal. Sensor World :03

10. Dai X, Ji C, Wang H (2019) Application of EXCEL commonly used in navigation data processing. IOP Conference Series: Materials Science and Engineering 569:052094. https://doi.org/10.1088/1757-899x/569/5/052094

11. Eggert P (2005) Text-encoding, Theories of the Text, and the 'Work-Site.' Literary and Linguistic Computing 20(4):425-435. https://doi.org/10.1093/llc/fqi050.

12. Busch JE, Lin AD, Graydon PJ, Caudill M (2006) Ontology-based parser for natural language processing. U.S. Patent 7,027,974,B1, 11 April 2006.

13. Yang M, He W, Zhang Z, Xu Y, Yang H, Chen Y, Xu X (2019) An efficient storage and service method for multi-source merging meteorological big data in cloud environment. EURASIP Journal on Wireless Communications and Networking 2019(1):1-12

14. Sugimoto N, Shimizu A, Nishizawa T, Jin Y (2019) Long-range transport of mineral dust observed with the Asian Dust and aerosol lidar observation Network (AD-Net). In: E3S Web of Conferences. EDP Sciences, p 02001

15. Leblanc T, Sica RJ, van Gijsel JA, Haefele A, Payen G, Liberti G (2016) Proposed standardized definitions for vertical resolution and uncertainty in the NDACC lidar ozone and temperature algorithms-Part 3: Temperature uncertainty budget. Atmospheric Measurement Techniques 9(8):4079_ 4101

16. Shenghua X, Xianghong W, Jiping L, Yi Y, An L, Mengmeng L (2020) Dynamic Visualization of Spatio-Temporal Process Model Based on NetCDF and Optimal Interpolation for Marine Environment. Environmental Engineering and Management Journal 19:1957-1967

17. Fernald FG (1984) Analysis of atmospheric lidar observations: some comments. Applied optics 23(5):652-653

18. Sugimoto N, Huang Z, Nishizawa T, Matsui I, Tatarov B (2012) Fluorescence from atmospheric aerosols observed with a multi-channel lidar spectrometer. Optics Express 20(19):20800-20807

19. Zhao Y, Wang X, Cai Y, Pan J, Yue W, Xu H, Wang J (2021) Measurements of atmospheric aerosol hygroscopic growth based on multi-channel Raman-Mie lidar. Atmospheric Environment 246:118076. https://doi.org/10.1016/j.atmosenv.2020.118076

20. Lin C-W, Hong T-P, Lu W-H (2011) An effective tree structure for mining high utility itemsets. Expert Systems with Applications 38(6):7419-7424

21. Singh H, Sharma R (2012) Role of adjacency matrix \& adjacency list in graph theory. International Journal of Computers \& Technology 3(1):179-183

22. Samelin K, Pöhls HC, Bilzhause A, Posegga J, de Meer H (2012) On Structural Signatures for Tree Data Structures. In: Bao F, Samarati P, Zhou J (eds) Applied Cryptography and Network Security. Springer Berlin Heidelberg, Berlin, Heidelberg, pp 171-187

23. Krijnen T, Beetz J (2020) An efficient binary storage format for IFC building models using HDF5 hierarchical data format. Automation in Construction 113:103134. https://doi.org/10.1016/j.autcon.2020.103134

24. Belov V, Tatarintsev A, Nikulchev E (2021) Choosing a Data Storage Format in the Apache Hadoop System Based on Experimental Evaluation Using Apache Spark. Symmetry-Basel 13(2):195. https://doi.org/10.3390/sym13020195

25. Nikulchev E, Ilin D, Gusev A (2021) Technology Stack Selection Model for Software Design of Digital Platforms. Mathematics 9(4):308. https://doi.org/10.3390/math9040308 
26. Grasberger H, Duprat J-L, Wyvill B, Lalonde P, Rossignac J (2016) Efficient data-parallel tree-traversal for BlobTrees. Computer-Aided Design 70:171-181. https://doi.org/10.1016/j.cad.2015.06.013

27. Shichkina Y, Kupriyanov M, Shevsky V (2018) The Application of Graph Theory and Adjacency Lists to Create Parallel Queries to Relational Databases. In: Galinina O, Andreev S, Balandin S, Koucheryavy Y (eds) Internet of Things, Smart Spaces, and Next Generation Networks and Systems. Springer International Publishing, Cham, pp 138-149

28. Andrusky K, Curial S, Amaral JN (2007) Tree-Traversal Orientation Analysis. In: Almási G, Caşcaval C, Wu P (eds) Languages and Compilers for Parallel Computing. Springer Berlin Heidelberg, Berlin, Heidelberg, pp 220-234

29. Wang Y, Tang L, Zhang J, Gao T, Wang Q, Song Y, Hua D (2018) Investigation of Precipitable Water Vapor Obtained by Raman Lidar and Comprehensive Analyses with Meteorological Parameters in Xi'an. Remote Sensing 10(6). https://doi.org/10.3390/rs10060967 